\title{
Comunicação
}

[Comunication]

\section{Aspecto médico veterinário legal das lesões em aves silvestres promovidas por colisões com aeronaves}

\author{
[Medico-legal veterinary aspect of lesions in bird strike]
}

\author{
L.T.R. Silva ${ }^{1}$, J.S.A. Silva ${ }^{2}$, R.F.S. Regueira ${ }^{2}$, V.P.M. Rolim ${ }^{1}$, M.R. Barros ${ }^{1}$, \\ A.A.F. Oliveira ${ }^{1 *}$ \\ ${ }^{1}$ Universidade Federal Rural de Pernambuco - UFRPE - Recife, PE \\ ${ }^{2}$ Centro de Apoio ao Desenvolvimento Tecnológico - CDT-UnB - Brasília, DF
}

Os sítios aeroportuários são frequentados por diversas espécies de aves. Em Pernambuco, no Aeroporto Internacional do Recife/Guararapes Gilberto Freyre, considerado o terceiro aeroporto nordestino em número de colisões registradas (Novaes e Alvarez, 2010), foram identificadas mais de 60 espécies de aves, porém algumas se destacam em relação à segurança aeronáutica por estarem envolvidas em episódios de colisões, tais como urubu-de-cabeça-preta (Coragyps atratus), quero-quero (Vanellus chilensis), carcará (Caracara plancus), entre outras (Anuário..., 2014).

Cerca de $90 \%$ das colisões de aeronaves com aves ocorrem nas fases de corrida de pouso e decolagem (Johnson, 2011). As lesões resultantes estão diretamente relacionadas ao momento do impacto entre ave/aeronave. Segundo Silva (2011), a força de impacto de uma ave de $2 \mathrm{~kg}$ com uma aeronave a $300 \mathrm{~km} / \mathrm{h}$ pode chegar a sete toneladas, sendo as consequências imprevisíveis.

Nesse sentido, a medicina veterinária legal depara-se com áreas consideradas como excepcionais ou imprevistas, como, por exemplo, a análise de cadáveres oriundos de colisões com aeronaves. Tal avaliação possibilita a identificação de espécies e a caracterização de lesões, o que contribui significativamente para a análise de risco que as espécies representam à indústria da aviação (Johnson, 2011), bem como norteia a elaboração e a efetivação de ações que objetivem a redução do risco aviário (Novaes e Alvarez, 2010).
Dessa forma, objetivou-se analisar, sob o aspecto médico veterinário legal, lesões em aves silvestres vítimas de colisão com aeronaves procedentes do Aeroporto Internacional do Recife/Guararapes Gilberto Freyre, Pernambuco.

Para execução do estudo, previamente aprovado pelo Ceua/UFRPE (Licença n. 078/2016), foram analisados cadáveres de quatro aves das espécies Caracara plancus (carcará) $(\mathrm{n}=1)$ e Vanellus chilensis (quero-quero) $(\mathrm{n}=3)$, com histórico de colisão registrado, encontrados na área da pista de pouso e decolagem do Aeroporto Internacional do Recife/Guararapes Gilberto Freyre, Pernambuco. As aves recolhidas pela equipe responsável foram prontamente armazenadas em freezer a $-20^{\circ} \mathrm{C}$ e posteriormente encaminhadas à área de Patologia do Departamento de Medicina Veterinária da UFRPE, para realização do exame necroscópico. A primeira ave analisada era um espécime de $C$. plancus, em boa condição corporal, apresentando ao exame externo: lesão cortocontusa determinando amputação traumática em asa direita (Fig. 1A), lesão contusa do tipo fratura completa fechada em fêmur distal direito e fratura transversal em osso frontal (Fig. 1B), com presença de infiltração hemorrágica no foco da fratura. Ao exame interno, evidenciou-se presença de fratura completa na terceira e na quarta costela esquerda, com presença de hematoma adjacente (Fig. 1C) e hemorragia difusa em cavidade celomática. Na moela, foi observada a presença de fragmentos de ossos (Fig. 1D). 


\section{Silva et al.}
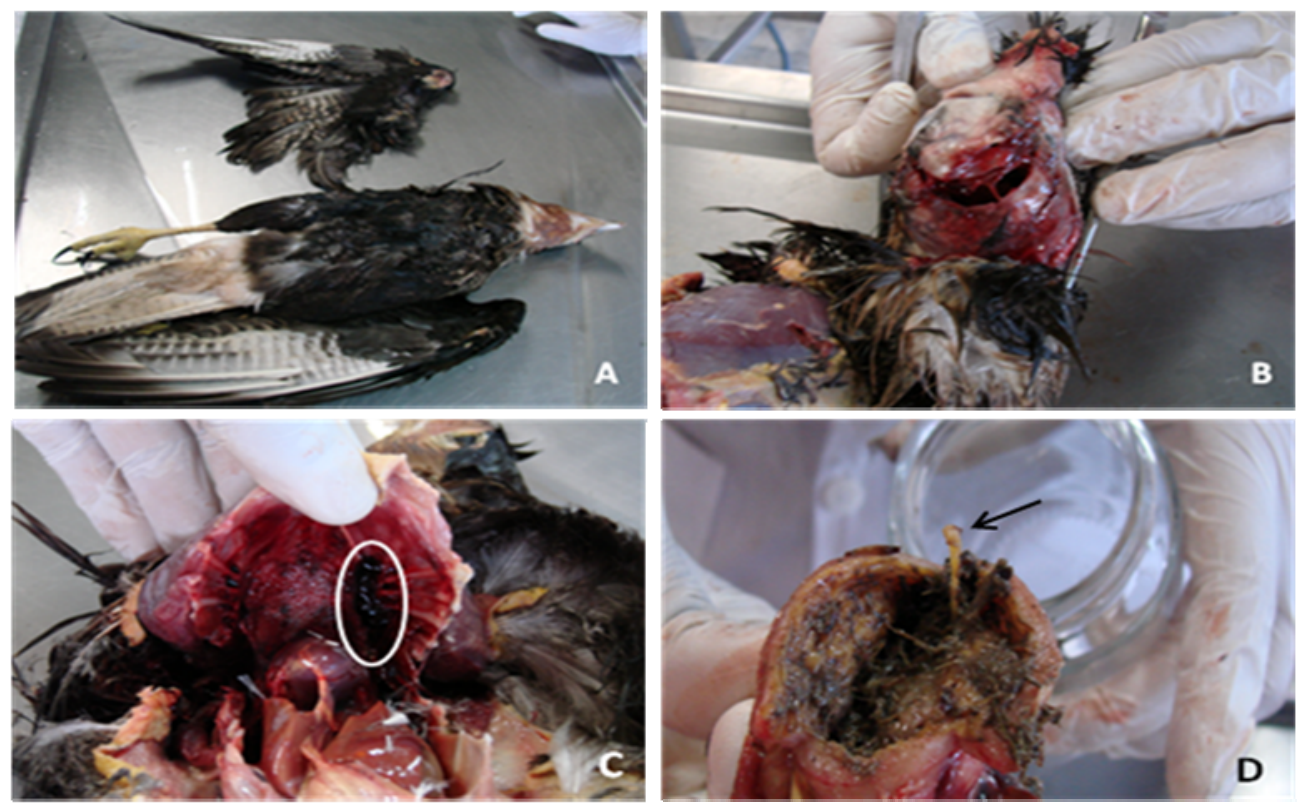

Figura 1. Caracara plancus. A) Amputação traumática da asa direita. B) Fratura transversal em osso frontal. C) Vista ventral da cavidade celomática. Hematoma (círculo) adjacente às costelas esquerdas. D) Fragmento ósseo (seta) em moela.

As demais aves analisadas (segunda, terceira e quarta) eram espécimes de $V$. chilensis. Em todas as aves, constatou-se boa condição corporal. $\mathrm{Na}$ segunda ave (Fig. 2A), detectou-se, ao exame externo, lesão cortocontusa com secção

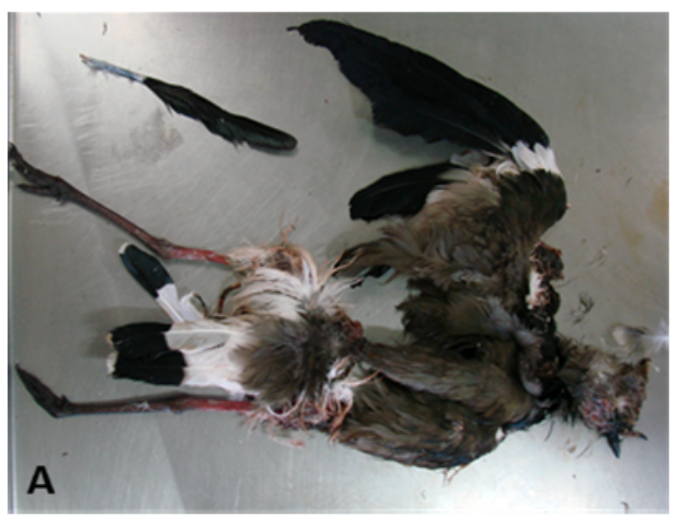

transversal completa, separando a cavidade celomática em dois segmentos (Fig. 2B), além de fratura craniana transversal em osso frontal. $\mathrm{O}$ exame interno foi prejudicado pela evisceração traumática no momento da colisão.

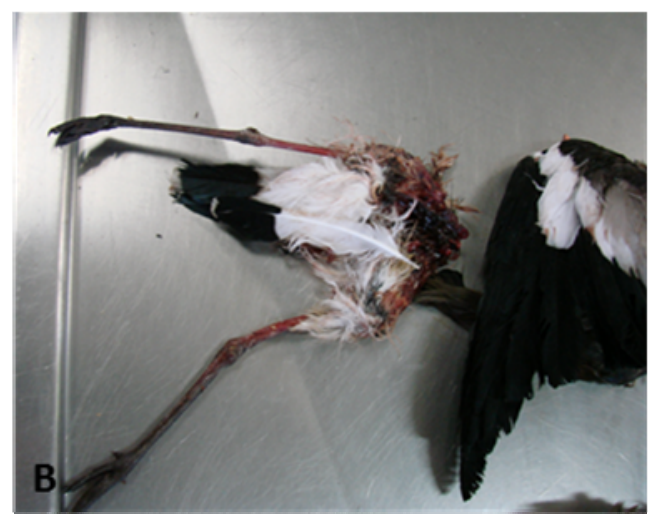

Figura 2. Vanellus chilensis. A) Visão geral da ave. B) Detalhe da lesão cortocontusa em plano ventral da ave.

Na terceira ave analisada (Fig. 3A), da espécie $V$. chilensis, constatou-se, ao exame externo, lesão contusa do tipo fratura horizontal medial direita nos ossos temporal e parietal (Fig. 3B), hemorragia intracraniana, proptose traumática unilateral direita (Fig. 3C), fratura completa fechada no úmero esquerdo. Ao exame interno, evidenciou-se a presença de lesão contusa do tipo fratura completa em esterno (Fig. 3D), bem como de hemorragia difusa em cavidade celomática (Fig. 3E). 

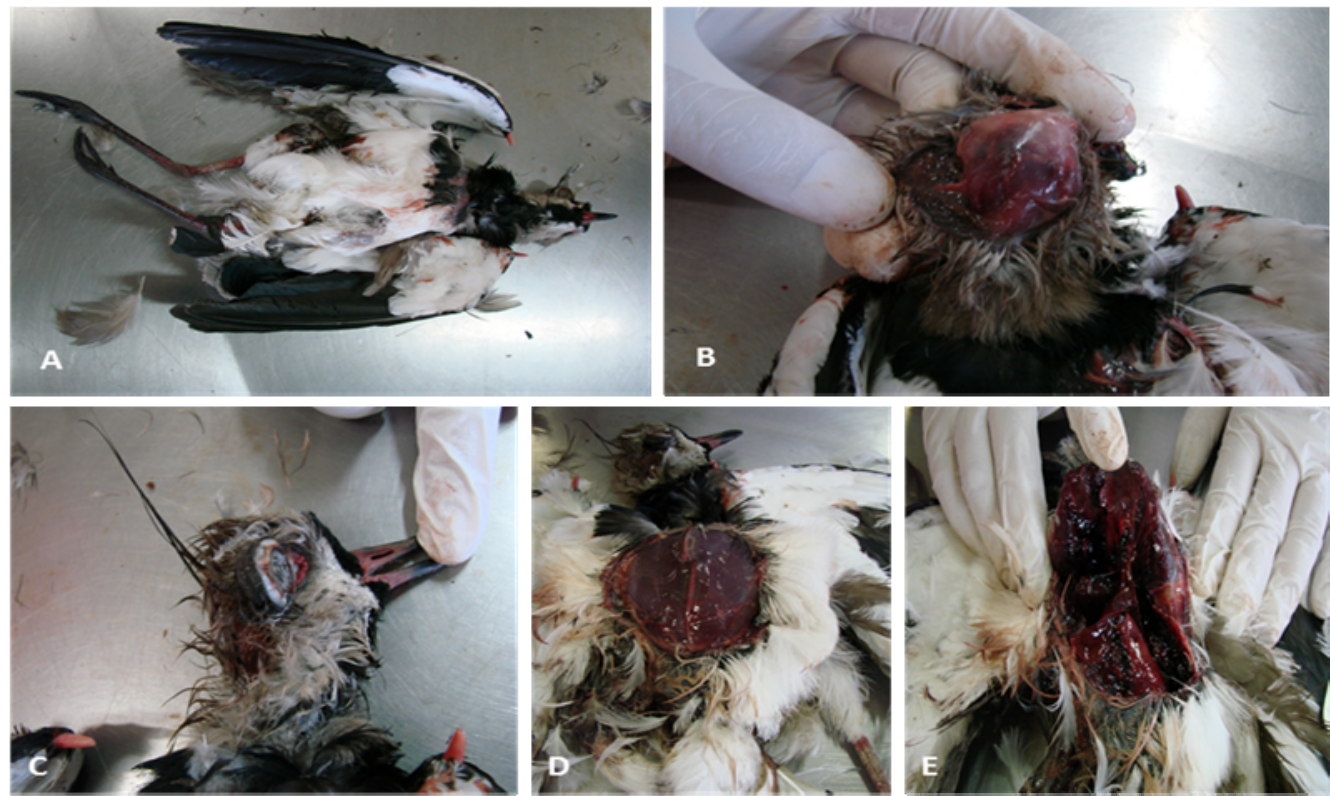

Figura 3. Vanellus chilensis. A) Visão geral da ave. B) Lesão contusa do tipo fratura horizontal medial direita nos ossos temporal e parietal. C) Proptose traumática unilateral direita. D) Lesão contusa do tipo fratura completa em esterno. E) Hemorragia difusa em cavidade celomática.

A quarta ave necropsiada da espécie $V$. chilensis (Fig. 4A), ao exame externo, não apresentou lesões detectáveis. Ao exame interno, após o rebatimento da pele, constatou-se a presença de equimoses em região ventral ao osso parietal (Fig. 4B) e hematoma peri-hepático associado à hemorragia na cavidade celomática (Fig. 4C). A análise do sistema digestório revelou a presença de coleópteros preenchendo por completo a moela (Fig. 4D).

Quanto ao aspecto médico veterinário legal, as lesões, totalizando 20, foram categorizadas de acordo com o exposto na Fig. 5.
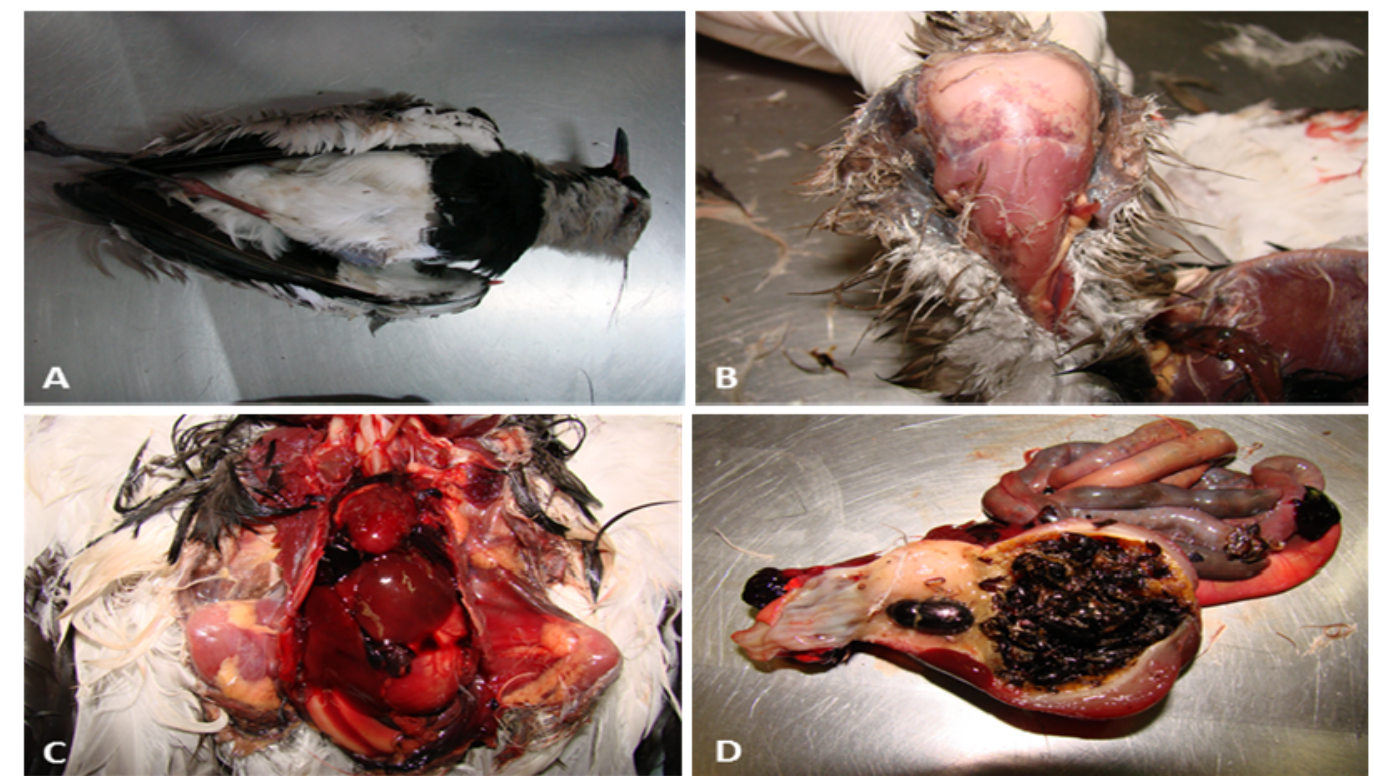

Figura 4. Vanellus chilensis. A) Visão geral da ave. B) Equimoses em região ventral ao osso parietal. C) Hematoma peri-hepático. D) Coleópteros no interior da moela. 


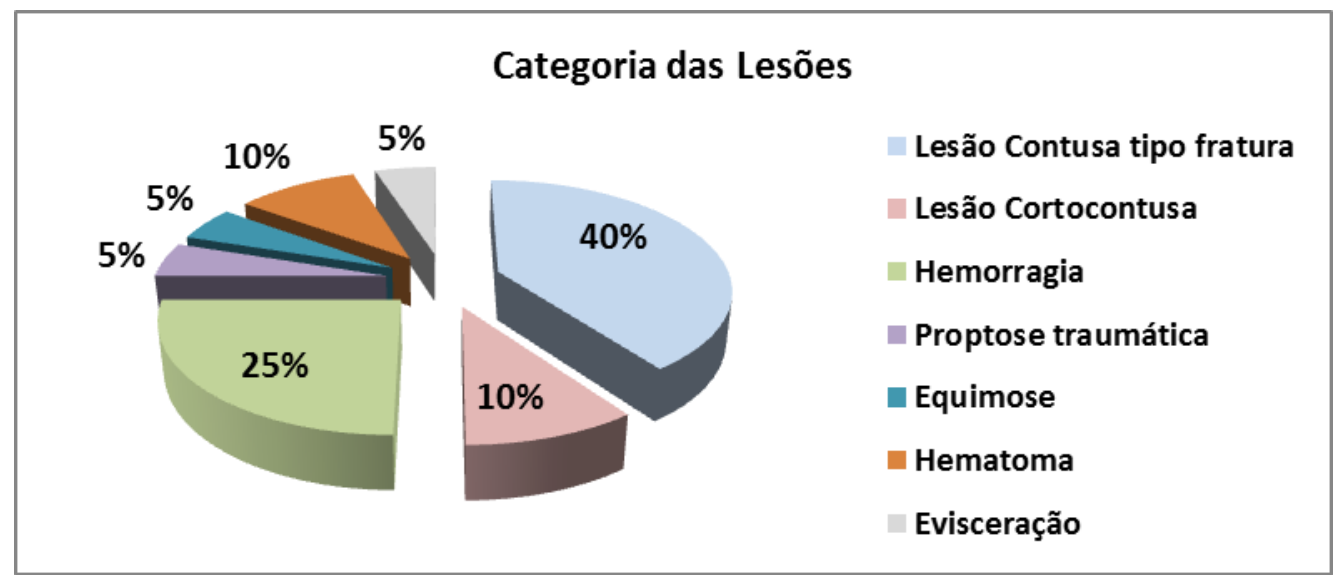

Figura 5. Análise qualitativa e efetivo de lesões categorizadas sob o aspecto médico veterinário legal em aves vítimas de colisão com aeronaves.

A presença de aves nos sítios aeroportuários consiste em problema nacional de grandes dimensões, visto que, associada ao aumento do tráfego aéreo, eleva consideravelmente o risco de colisões (Mendonça, 2009). De forma similar aos demais aeroportos do país, o Aeroporto Internacional do Recife/Guararapes Gilberto Freyre enfrenta a mesma problemática, contextualizada na presente comunicação pela análise de quatro aves com óbito confirmado em decorrência de colisão com aeronaves.

As aves analisadas no estudo, das espécies $V$. chilensis e C. plancus, são relatadas como adaptadas aos centros urbanos e frequentemente relacionadas a colisões com aeronaves (Mendonça, 2009; Guedes et al., 2010; Cardoso et al., 2014); desse modo, são consideradas como um risco à aviação (Anuário..., 2014).

Cardoso et al. (2014) observaram que as espécies $V$. chilensis apresentam o comportamento de pousar na pista de pouso e decolagem, em contraste com $C$. plancus, que tende a cruzar a pista. A análise comportamental das aves no sítio aeroportuário é essencial no entendimento das lesões apresentadas no momento da análise necroscópica. As lesões traumáticas evidenciadas nos casos analisados relacionam-se diretamente à velocidade da aeronave e à massa da ave, o que justificaria a presença de politraumatismos, comprovados neste estudo em todas as aves, que podem variar qualitativa e quantitativamente (Guedes et al., 2010). Este fato foi perceptível, por exemplo, na segunda ave da espécie $V$. chilensis, que apresentou separação da cavidade celomática em dois segmentos. Vale ressaltar que, conforme Sheehy et al. (2005), torna-se difícil categorizar e resumir uma análise post mortem de aves procedentes de colisão, em virtude da quantidade de dados qualitativos observados.

A predominância de lesões contusas nas aves deste estudo, caracterizadas pela presença de fraturas, equimoses e hematomas, justificam-se pela ação contundente promovida pelo tipo de agente vulnerante envolvido (aeronaves). As fraturas cranianas evidenciadas são relatadas nos escassos estudos de caracterização de lesões decorrentes de colisões entre aeronaves e aves. Lyne et al. (1998) detectaram fraturas cranianas em $37 \%$ das aves, do total de 92 necropsiadas, e Sheehy et al. (2005) mencionaram que, de 21 análises necroscópicas em aves de 25 espécies distintas vítimas de colisão com aeronaves, incluindo a espécie $V$. chilensis, $18,2 \%$ apresentaram lesões graves na cabeça, no pescoço e lesões vertebrais, sendo essas últimas não evidenciadas neste estudo. Segundo Merck (2007), traumas evidenciados em região de cabeça devem ser categorizados como procedentes de impacto ou aceleração/desaceleração e comumente revelam fraturas, hemorragias e hematomas, o que foi claramente percebido nas aves deste estudo. Entretanto, como anteriormente citado, a gravidade dessas lesões está diretamente relacionada ao peso da ave e à velocidade de impacto, fatores que podem ter interferido, por exemplo, na ausência de fraturas na quarta ave necropsiada. 
Em associação à fratura craniana observada na terceira ave analisada, foi evidenciada proptose traumática. De acordo com Merck (2007), traumas oculares são primariamente relacionados a lesões contusas na cabeça, sendo às vezes os únicos achados observados nessa condição.

As fraturas em ossos dos esqueletos axial, descritas em costelas e esterno, e apendicular no caso de úmero e fêmur, são condizentes ao tipo de agente vulnerante. Lyne et al. (1998) relataram a presença de fraturas em costela e esterno em 40,2\% do total de 92 aves necropsiadas, resgatadas no aeroporto de Dublin, na Irlanda, assim como a presença de fratura em membros em $90 \%$ das aves. As aves atingidas durante o voo podem expor as asas, em posição aberta, ao impacto direto, com potenciais agravantes à sua estrutura.

As lesões cortocontusas, observadas na amputação traumática da primeira ave e na separação da cavidade celomática na segunda ave, caracterizaram-se por apresentarem forma variável, bordos irregulares e contundidos e ausência de pontes de tecidos íntegros. As turbinas das aeronaves, constituídas por hélices, movimentam grandes massas de ar, e supõe-se que gerem uma espécie de força de sucção em relação à ave, determinando, desse modo, o aparecimento de amputações traumáticas e fragmentação do corpo com evisceração. Em relação à evisceração, Lyne et al. (1998) consideraram como sendo um dos traumas elencados para o estabelecimento do que chamaram de "Bird Strike Syndrome", composta também por fraturas múltiplas em membros, lesões abdominais e torácicas graves, culminando em hemorragias cavitárias, de acordo com o observado neste estudo.

Hematomas e equimoses são considerados lesões contusas e relacionam-se diretamente ao agente vulnerante (contundente) que as produziu. A presença de hematomas remete ao rompimento de vasos calibrosos sem a difusão na malha tecidual, diferentemente das equimoses, que se caracterizam pela ruptura de vasos menos calibrosos com difusão na malha tecidual. Para que as equimoses sejam verificadas, há a necessidade da presença de um plano mais resistente abaixo da região traumatizada (França, 2005), conforme constatado em região ventral ao osso parietal na quarta ave necropsiada.

Ao exame da moela, constatou-se a presença de fragmentos ósseos em espécime de Caracara plancus e de coleópteros em Vanellus chilensis, indicativo de que os aeroportos possuem fatores atrativos, tais como alimento, para uma diversidade de animais, incluindo as aves, o que eleva a probabilidade de colisões com aeronaves.

A análise sob o aspecto da medicina veterinária legal de animais resgatados em sítios aeroportuários é essencial, pois, além da tentativa de estabelecimento de um padrão que permita diferenciar as lesões ocasionadas por aeronaves, norteia as medidas de gerenciamento de risco de fauna, uma vez que, ao exame necroscópico, é possível identificar a espécie envolvida ou ao menos obter material biológico para identificação.

Palavras-chave: aeroporto, Caracara plancus, Vanellus chilensis, risco aviário

\begin{abstract}
The objective of this study was to describe lesions in four birds, victims of collisions with aircraft, coming from the International Airport of Recife/Guararapes Gilberto Freyre, Pernambuco, Brazil. Contused wounds were predominant in birds in this study, characterized by the presence of fractures, bruises and hematomas and justified by the blunt action promoted by the type of vulnerant agent involved (aircraft). The analysis under medico-legal veterinary aspect of lesions in bird strike is essential, because in addition to the attempt to establish a standard for differentiating the aircraft lesions, it can guide the bird strike hazard management programs at airports, since the necropsy allows the identification of the species involved or at least facilitates the obtaining biological material for identification.
\end{abstract}

Keywords: airport, Caracara plancus, Vanellus chilensis, aircraft strike 


\section{REFERÊNCIAS}

ANUÁRIO de risco de fauna. Panorama estatístico. Cidade: CENIPA, 2014. Disponível em: <http://www.cenipa.aer.mil.br/> Acessado em: 08 ago. 2016.

CARDOSO, C.O.; GOMES, D.N.; SANTOS, A.G.S. et al. Risco de colisão de aves com aeronaves no aeroporto internacional de Parnaíba, Piauí, Brasil. Ornitol. Neotrop., v.25, p.179-193, 2014.

FRANÇA, G.V. Traumatologia médico-legal. In: (Ed.). Fundamentos de medicina legal. Rio de Janeiro: Guanabara Koogan, 2005. p.38108.

GUEDES, F.L.; BRAND, D.H.; LINHARES, B.P.; PAIVA, L.V. Avifauna relacionada ao risco de colisões aéreas no Aeroporto Internacional Presidente Juscelino Kubitschek, Brasília, Distrito Federal, Brasil. Rev. Conex. SIPAER, v.2, p.230-243, 2010.

JOHNSON, R.N. Conservation genetics and wildlife forensics of birds. In: HUFFMAN, J.E.; WALLACE, J.R. (Eds.). Wildlife forensics: methods and applications. [New York]: John Wiley \& Sons, 2011. p.305-307.
LYNE, K.; GASSNER, I.; BOLGER, R.; KELLY, T.C. Is There a bird strike syndrome? Preliminary results from autopsy findings. Stara Lesna, Slovakia: International Bird Strike Committee, 1998. p.14-18.

MENDONÇA, F.A. Gerenciamento do perigo aviário em aeroportos. Rev. Conex. SIPAER, v.1, p.153-174, 2009.

MERCK, M.D. Patterns of non-accidental injury: non-penetrating Injuries. In: MERCK, M.D. (Ed.). Veterinary forensics: animal cruelty investigations. Ames: Blackwell Publishing, 2007. p.79-100.

NOVAES, W.G.; ALVAREZ, M.R.D.V. O perigo aviário em aeroportos do nordeste do Brasil: análise das colisões entre aves e aviões entre os anos de 1985 e 2009. Rev. Conex. SIPAER, v1, p.47-68, 2010.

SHEEHY, S.; KELLY, T.C.; FENNESSY, G.; O'CALLAGHAN, M.J.A.; BOLGER, R. Bird strike syndrome: towards developing an index of bird injury. Athens: International Bird Strike Committee, 2005.

SILVA, R.E. Perigo aviário. Carta de segurança operacional. 3.ed. Rio de Janeiro: ANAC, 2011. p.3-5. 